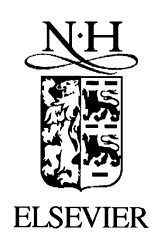

Nuclear Instruments and Methods in Physics Research A 457 (2001) 253-261

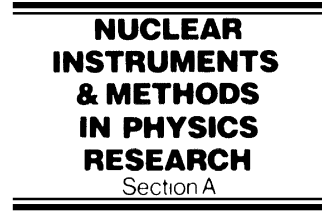

www.elsevier.n1/locate/nima

\title{
Gas proportional scintillation counters with a CsI-covered microstrip plate UV photosensor for high-resolution X-ray spectrometry
}

\author{
J.F.C.A. Veloso, J.M.F. dos Santos, C.A.N. Conde* \\ Departamento de Física, Universidade de Coimbra, P-3004-516 Coimbra, Portugal \\ Received 25 February 2000; received in revised form 12 June 2000; accepted 14 June 2000
}

\begin{abstract}
A xenon-filled gas proportional scintillation counter for X-ray spectrometry is described. The detector uses a microstrip plate covered with a thin CsI film as a photosensor rather than the usual photomultiplier tube. The achieved energy resolution for $5.9 \mathrm{keV} \mathrm{X}$-rays is $12 \%$ when the microstrip is operated within the xenon volume and $10.5 \%$ when the microstrip is operated in a P-10 environment, separated from the xenon volume by a quartz window. (C) 2001 Elsevier Science B.V. All rights reserved.
\end{abstract}

PACS: $07.85 . \mathrm{Nc} ; 29.40 . \mathrm{Cs} ; 29.40 . \mathrm{Mc} ; 85.60 . \mathrm{Ha}$

Keywords: Gas proportional scintillation counter; MSGC; CsI photocathode; X-rays

\section{Introduction}

Since the introduction of the first Gas Proportional Scintillation Counters (GPSCs) [1] they have been used for X-ray spectrometry in many applications such as X-ray astronomy, medical instrumentation and high-energy physics [2-5]. In particular, they combine room-temperature operation with relatively good energy resolution, large detection areas and high counting rates with reduced space charge effects, when compared with conventional proportional counters [2,5].

\footnotetext{
* Corresponding author. Tel.: + 351-2394-10654; fax: + 3512398-29158.

E-mail address: canconde@gian.fis.uc.pt (C.A.N. Conde).
}

The theory and operation of GPSCs have been described in detail in earlier publications [2,5]. Incident X-rays interact mostly in the drift region (Fig. 1) where the resulting primary electron cloud drifts under the influence of a weak electric field into a region with a stronger electric field, the scintillation region. Upon crossing the scintillation region, the primary electrons gain from the electric field enough energy to excite but not to ionise the gas media producing a light pulse as a result of the gas atoms de-excitation, which is proportional to the number of primary electrons and so to the X-ray energy. The statistical fluctuations associated to the light amplification processes are negligible compared to the ones associated to the primary electron cloud formation, resulting in a detector with a resolution approaching the fluctuations in the absorption process. 


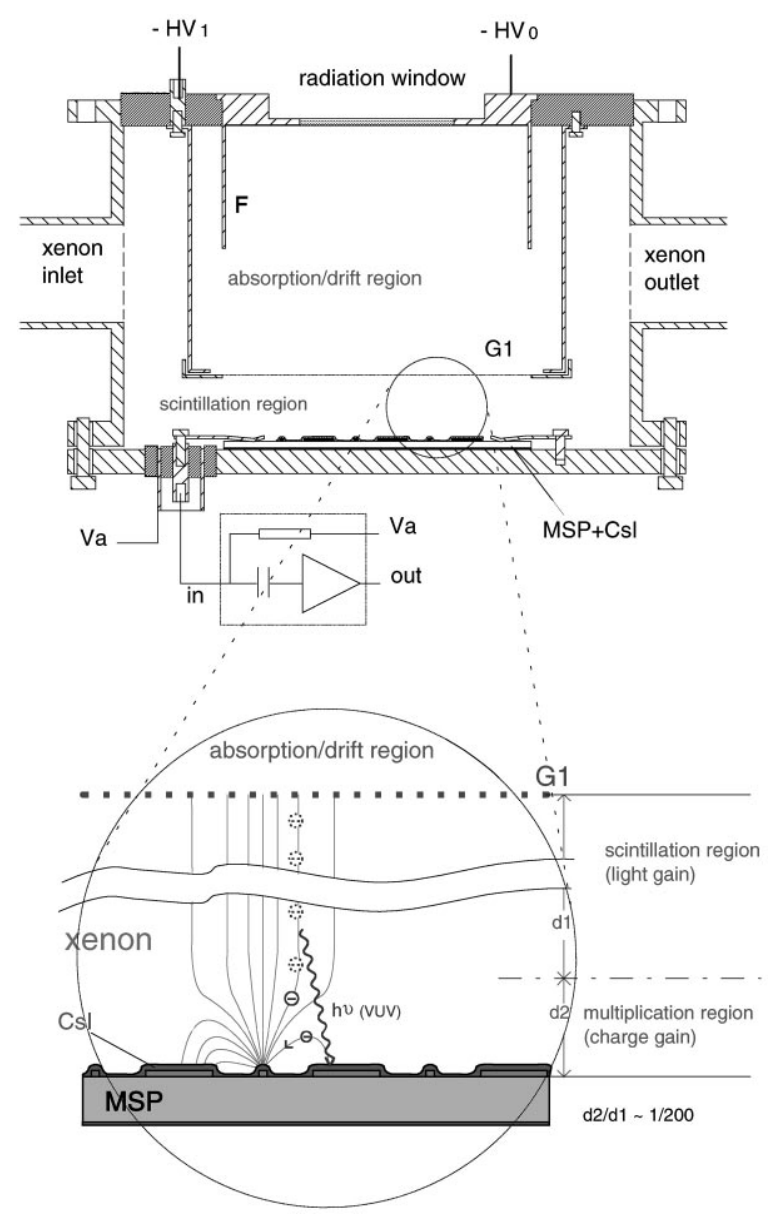

Fig. 1. Schematic of GPSC with integrated CsI-covered MSP photosensor.

A typical GPSC uses pure xenon as the filling gas and a quartz window photomultiplier tube (PMT) to detect the VUV electroluminescence produced in the scintillation region. The PMT not only limits the uniformity of the detection area of GPSCs but also increases their cost, power consumption, complexity, fragility and bulkiness, presenting a drawback in applications to areas such as portable devices for energy-dispersive X-ray fluorescence analysis, Mössbauer spectroscopy and operation under strong magnetic fields.

Alternatives to replace the PMT in a GPSC by a more convenient photosensor have been investigated [6-11]. Typically, these have been independent devices coupled to the GPSC by a VUV window. However, integrated photosensors that could be placed inside the GPSC envelope [12-14], in direct contact with the gas, are of great interest. The importance of the development of such integrated photosensors for RICH (ring-imaging Cherenkov) detectors, placed in direct contact with the VUV carrier gas, was also described [15-17]. They avoid the use of an interface VUV window that can be expensive, difficult to handle and can absorb the scintillation light.

Recently, the feasibility of detecting UV light with a thin layer of CsI photocathode, deposited directly on to a microstrip plate (MSP), was demonstrated [18,19].

The potential use of a CsI covered MSP as an integrated photosensor substituting the PMT in a GPSC was recognised, but the performance of the GPSCs based on that integrated photosensor was marginal, achieving an energy resolution of $17 \%$ for $5.9 \mathrm{keV}$ [13], worse than the one achievable with a conventional proportional counter. However, the substitution of the PMT and the elimination of the scintillation window is, in itself, a compelling reason for further development of such detectors and efforts for improvement are needed.

In this work we report improved experimental results obtained with a GPSC using a CsI-covered MSP as the VUV photosensor readout, in substitution of the PMT. We resume the research performed with the GPSC/CsI-MSP presented in Ref. [13], after overcoming the former limitations, improving the detector operating conditions and improving the MSP and CsI cleaning and handling procedures.

\section{Description}

Microstrip gas chambers (MSGC) [20] are miniaturised planar variants of the multiwire proportional chamber (MWPC): thin metal strips are photolithographed onto an insulating substrate with very small spacing between alternated anodes and cathodes. A low voltage applied between the closely spaced electrodes produces the intense electric field, around the anodes, required for charge multiplication in the gas medium. On the other hand, the closely spaced electrodes allow a rapid removal of the residual positive ions resulting from the electron 
avalanche. Thus, the MSP design results in a very compact detector with reduced space charge effects, relatively high gain and high counting rate capabilities, in addition to inherent spatial sensitivity.

When the MSP is covered with CsI, the incident VUV photons induce the emission of photoelectrons from the active areas, the cathode strips. The photoelectrons drift towards the anode strips producing charge avalanches in the intense electric field.

The CsI-covered MSP is placed within the xenon envelope, playing simultaneously two different roles (Fig. 1): it serves as the GPSC collection grid for the primary electron cloud (the second grid of the scintillation region); and serves as the amplification stage for the photoelectrons produced due to the VUV scintillation. The upper region, $d_{1}$, functions as the uniform field scintillation region of a conventional GPSC, while the behaviour of region $d_{2}(<50 \mu \mathrm{m}$ [21] $)$ is that of a standard MSGC. It was demonstrated that such a system functions as a GPSC rather than a MSGC [13]. However, a poor energy resolution was achieved.

The energy resolution, $R$, of a conventional GPSC is determined by the statistical fluctuations in the primary ionisation processes, in the production of VUV scintillation light and in the photosensor performance and can be given by [22]:

$$
R=2.35 \sqrt{\frac{F}{N}+\frac{1}{N}\left(\frac{J}{N_{\mathrm{s}}}\right)+\frac{1}{N_{\mathrm{e}}}\left(1+\left(\frac{\sigma_{q}}{G_{q}}\right)^{2}\right)}
$$

where $N$ is the average number of primary electrons produced per incident $\mathrm{X}$-ray photon, $F$ is the relative variance of $N$, the Fano factor, $N_{\mathrm{s}}$ is the average number of scintillation photons produced per primary electron, $J$ is the relative variance of $N_{\mathrm{s}}, N_{\mathrm{e}}$ is the average number of photoelectrons produced in the photosensor per incident X-ray photon and $\left(\sigma_{q} / G_{q}\right)^{2}$ is a measure of the fluctuations in the electron multiplication gain of the photosensor. The second term of Eq. (1) can be neglected since $J / N_{\mathrm{s}} \ll F$. For a $\operatorname{MSGC}\left(\sigma_{q} / G_{q}\right)^{2}$ is less than the $f$ parameter of standard proportional counters [23], which is $f \cong 0.6$ for xenon [23], while for a PMT $\left(\sigma_{q} / G_{q}\right)^{2} \approx 1[24]$.

Taking into account that $N=E_{x} / W$, the X-ray photon energy, $E_{x}$, divided by the mean energy to produce a primary electron, $W$, and defining the number of photoelectrons produced per primary electron, $L=N_{\mathrm{e}} / N[6]$, the FWHM $=R \times E_{x}$ of a GPSC will be given by

$$
\begin{aligned}
\text { FWHM } & =2.35 \sqrt{E_{x} \times W\left(F+\frac{1+f}{L}\right)} \\
& \Rightarrow \text { GPSC } / \text { MSGC } \\
\text { FWHM } & =2.35 \sqrt{E_{x} \times W\left(F+\frac{2}{L}\right)} \\
& \Rightarrow \text { GPSC } / \text { PMT }
\end{aligned}
$$

when using a MSGC (2) or a PMT (3) as the photosensor.

In Fig. 2 we plot FWHM as a function of $E_{x}$ for different $L$ values and for a GPSC/MSGC or /PMT combination, assuming $f=0.45$ for the MSGC [23], $W=22 \mathrm{eV}$ [25] and $F=0.17$ [26]. As seen, for the same X-ray photon energy, $E_{x}$, and for the same $L$ parameter, the theoretical limit for the energy resolution of a GPSC/MSGC combination would be lower than the one of a GPSC/PMT combination. However, the efficiency of a photosensor to convert VUV light into photoelectrons,

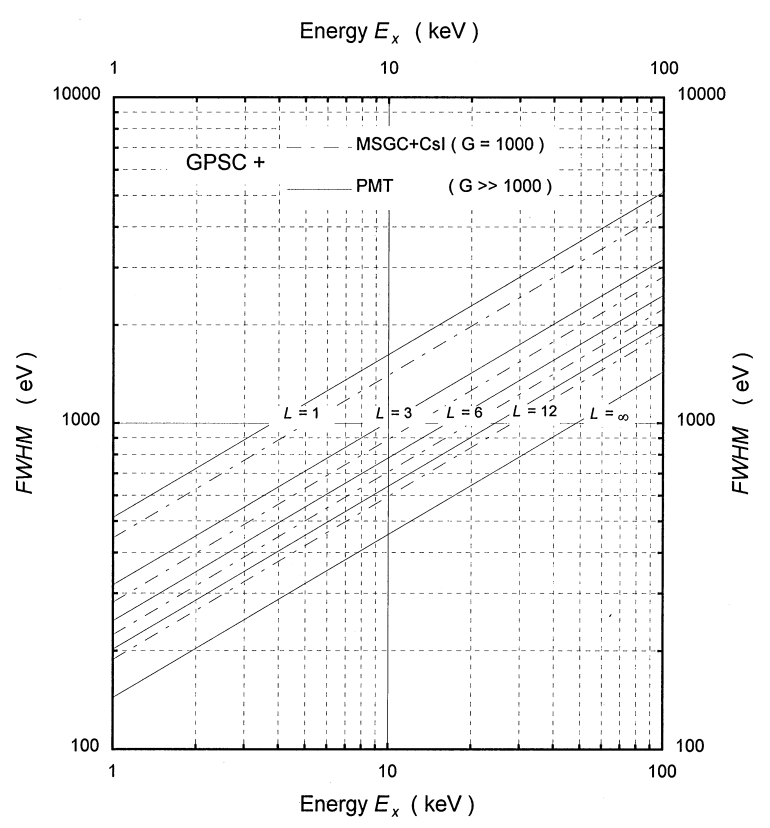

Fig. 2. Calculated FWHM of the GPSC pulse-height distribution as a function of the X-ray energy for a GPSC/PMT and for a GPSC/MSGC combination. 
related with the $L$ parameter, must be taken into account. Since the $f$ parameter of MSGCs is smaller than in PCs [23], the theoretical limit for the energy resolution of the GPSC/MSGC combination will still be better than GPSC/PC combination.

\section{Experimental set-up and procedures}

The design of the detector used in this work is depicted schematically in Fig. 1 . The thin, aluminised kapton window and the focusing electrode $F$ are maintained at negative high voltage, $-H V_{0}$, while the G1-grid $(80 \mu \mathrm{m}$ diameter stainless-steel wire with $900 \mu \mathrm{m}$ spacing) and its holder electrode are kept at $-H V_{1}$. The MSP is a CERN model MS-4. It consists of $10 \mu \mathrm{m}$ anodes and $80 \mu \mathrm{m}$ cathodes with a $200 \mu \mathrm{m}$ pitch, fabricated of $0.2 \mu \mathrm{m}$ thick chromium film deposited on a $500 \mu \mathrm{m}$ Desag D263 glass substrate. The backplane is a flat, unstructured layer of $0.1 \mu \mathrm{m}$ chromium. The backplane and cathodes are maintained at ground potential while a positive voltage $V_{\mathrm{a}}$ is applied to all the anodes. The voltage difference in the scintillation region is approximately $H V_{1}$. A $500 \mathrm{~nm}$ thick and $30 \mathrm{~mm}$ in diameter layer of high-purity CsI was vacuum deposited onto the surface of the MSP. The detector was filled with xenon at 800 Torr.

The detector design is similar to the one described in Ref. [13]. The depth of the scintillation region was increased by $25 \%$, up to $10 \mathrm{~mm}$, and the distance between the G1 holder electrode and the detector body was enlarged for improved electrical insulation. With these changes the scintillation light produced for the same reduced electric field (electric field intensity divided by the gas pressure, $E / p)$ was increased by $25 \%$ and reduced electric fields as high as $7 \mathrm{~V} \mathrm{~cm}^{-1} \mathrm{Torr}^{-1}$ could be achieved in the scintillation region. Since electrical insulation limits the maximum high voltage that could be applied before breakdown, a compromise had to be made between the scintillation region depth and the maximum reduced electric field to be achieved in this region. This compromise must also include the reduced electric field in the $4 \mathrm{~cm}$ deep absorption region and a value of $E / p=0.35 \mathrm{~V} \mathrm{~cm}^{-1}$ Torr $^{-1}$ was chosen during the tests, for best-detector performance.
Another and probably the most important improvement carried out in this work is related to the CsI film preparation. In the first prototype [13], the maximum $V_{\text {a }}$ voltage allowed before occurring breakdown in the CsI covered MSP was $275 \mathrm{~V}$, far below the one achieved for that same MSP without the CsI layer, which was about $500 \mathrm{~V}$. These voltages correspond to electron avalanche gains of 50 and 3000, respectively. The low gain then achieved was the limiting factor responsible for the marginal results obtained with that first prototype.

A possible explanation could be the water contamination of the CsI film. Thus, special care was taken to avoid this contamination: the MSP was heated at temperatures of about $100^{\circ} \mathrm{C}$ during $1 \mathrm{~h}$ before CsI evaporation, and the CsI film was heated under vacuum at about $80^{\circ} \mathrm{C}$ for $24 \mathrm{~h}$ after the CsI-covered MSP had been placed inside the detector. The heating of CsI under vacuum to reduce contamination was already referred to by other authors [27-29]. With this procedure, $V_{\text {a }}$ voltages above $390 \mathrm{~V}$ could be used and, in these new conditions, the positive photon feedback became the limiting factor responsible for maximum usable $V_{\mathrm{a}}$.

Anode pulses were pre-amplified with a CANBERRA 2006 charge-to-voltage preamplifier (sensitivity of $235 \mathrm{mV} / 10^{6}$ ion pairs), linearly amplified ( $5 \mu$ s time constants), and pulse-height analysed. For peak amplitude and energy-resolution measurements pulse-height distributions were fitted to a Gaussian superimposed on a linear background.

\section{Experimental results and discussion}

In Fig. 3 we depict the detector relative pulse amplitude as a function of $E / p$ in the scintillation region for 5.9 and $22.1 \mathrm{keV} \mathrm{X}$-rays, while maintaining the photosensor gain $\left(V_{\mathrm{a}}=340 \mathrm{~V}\right)$ constant. As seen, above the $\mathrm{Xe}$ scintillation threshold $\left(\sim 1 \mathrm{~V} \mathrm{~cm}^{-1} \mathrm{Torr}^{-1}\right)$ the linear behaviour characteristic of the secondary scintillation intensity [30] is observed. Below this threshold, the pulse amplitude is due to the charge amplification of the primary electrons in the microstrip. As all $5.9 \mathrm{keV}$ $\mathrm{X}$-rays interact in the absorption region, the number of their primary electrons that cross grid 


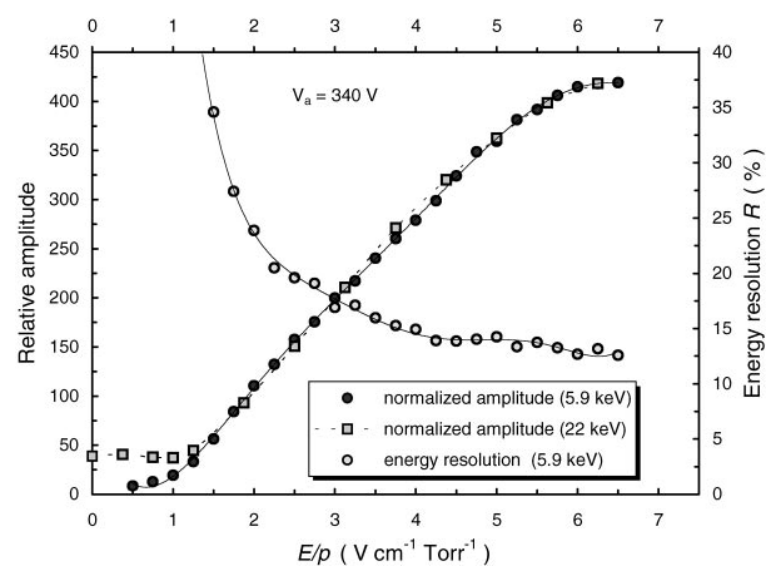

Fig. 3. GPSC relative pulse amplitude and energy resolution as a function of the reduced electric field in the scintillation region for 5.9 and $22.1 \mathrm{keV} \mathrm{X}$-rays and for a constant photosensor gain $\left(V_{\mathrm{a}}=340 \mathrm{~V}\right)$.

G1 and are collected in the MSP approaches zero as the electric field in the scintillation region becomes weaker [31]. However, for $22.1 \mathrm{keV}$ some of the X-rays do also penetrate in the scintillation region and thus, for these events, all primary electrons are collected and their pulse amplitude becomes constant for electric fields below the scintillation threshold.

For high electric fields, detector pulse amplitude deviates from the characteristic GPSC behaviour [30] and saturates. This trend can be explained if we take into account the electric field intensity at the CsI surface and its role in the extraction of the produced photoelectrons. It was shown [32] that for a CsI film in an argon environment, only for reduced electric field intensities above about $3 \mathrm{~V} \mathrm{~cm}^{-1} \mathrm{Torr}^{-1}$, the photoelectron collection efficiency reaches values close to the ones obtained for a CsI film placed under vacuum. In Fig. 4 we depict the reduced electric field intensities perpendicular to the CsI surface $\left(E_{y} / p\right)$ in the centre of the cathode strips as a function of the reduced electric field in the scintillation region for different $V_{\mathrm{a}}$ voltages. These results were obtained using a $2 \mathrm{D}$ electric field simulator. ${ }^{1}$ As seen from Fig. 4, the extraction of

\footnotetext{
${ }^{1}$ Maxwell 2D, Ansoft Corporation, Four Station Square, Suite 660, Pittsburgh, PA 15219, USA.
}

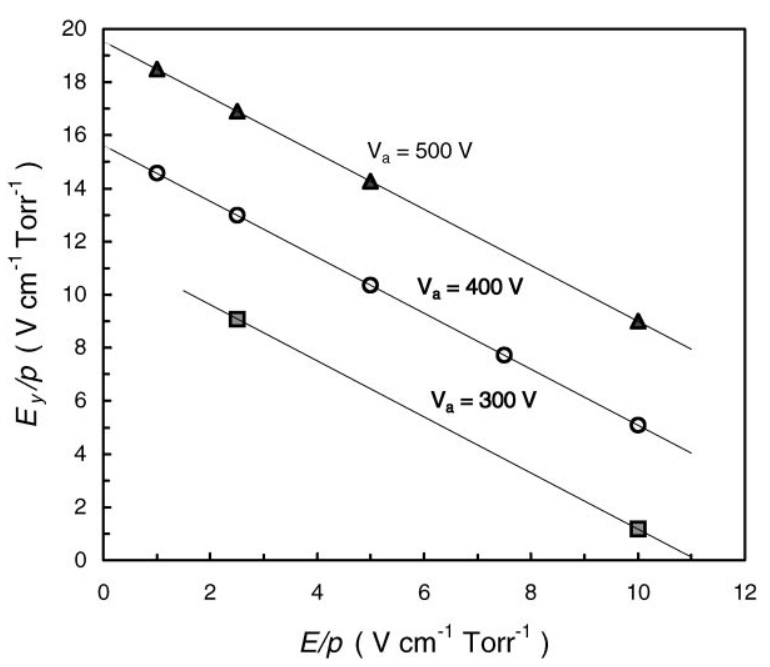

Fig. 4. Calculated reduced electric field component perpendicular to the MSP, $E_{y} / p$, at the centre of the cathode strips as a function of the reduced electric field in the scintillation region for different anode-to-cathode strips voltages.

photoelectrons from the CsI film may be jeopardised for high $E / p$ in the scintillation region since $E_{y} / p$ can reach values below $3 \mathrm{~V} \mathrm{~cm}^{-1} \mathrm{Torr}^{-1}$. Consequently, an increase of $E / p$ in this region may not lead to an increase of the detected photoelectrons. Fig. 4 also shows the importance of operating the photosensor at high $V_{\mathrm{a}}$ values in order to achieve good photoelectron collection efficiencies for the high electric fields in the scintillation region.

Fig. 3 also depicts the detector energy resolution as a function of $E / p$ in the scintillation region for $5.9 \mathrm{keV}$ X-rays and for $V_{\mathrm{a}}=340 \mathrm{~V}$. This resolution achieves best results for the highest $E / p$. Energy resolutions of $12 \%$ and $7 \%$ can be achieved for 5.9 and $22.1 \mathrm{keV} \mathrm{X}$-rays, respectively. Since the electrons, coming from the MSGC drift region (as is the case of primary electrons), have lower avalanche gains than the ones coming from the cathode strips [33], it is not possible to calculate the $L$ parameter for Eq. (2), from the amplitude gain presented in Fig. 3. However, if one considers the same avalanche gain for both primary electron and photoelectron, an upper limit of $L=10$ can be estimated from the depicted results for $22.1 \mathrm{keV}$; nevertheless we stress that a factor down to 0.1 between the two events is admissible [33]. From Eq. (2) and from the obtained energy resolution for $5.9 \mathrm{keV} \mathrm{X}$-rays we 
can estimate $L \sim 3$, which is much lower than $L \sim 14$ estimated from Eq. (3) for a GPSC/PMT combination with a typical $8.0 \%$ energy resolution at $5.9 \mathrm{keV}$. The $L$-value could be increased using a larger MSP and/or a better photocathode-stripsarea-to-total-MSP-area ratio. For our MSP the total area is only $30 \times 30 \mathrm{~mm}^{2}$ and the cathodestrips-area-to-total-MSP-area ratio is small ( $\sim 40 \%$ ) when compared to other MSPs already referred to in the literature (e.g. above 90\% [34]).

In Fig. 5 we depict the detector relative pulse amplitude and energy resolution as a function of $V_{\mathrm{a}}$ for $5.9 \mathrm{keV} \mathrm{X-rays,} \mathrm{while} \mathrm{maintaining} E / p$ in the scintillation region at $5.25 \mathrm{~V} \mathrm{~cm}^{-1} \mathrm{Torr}^{-1}$. A detector energy resolution of $12 \%$ is achieved for $V_{\mathrm{a}}=360 \mathrm{~V}$. An absolute photosensor gain of 700 is estimated for this $V_{\text {a }}$ voltage. Also, in Fig. 5, an exponential function (solid line) is superimposed on the experimental results. As expected, the photosensor gain depicts the characteristic exponential variation of charge avalanche processes in MSGCs. For $V_{\mathrm{a}}$ voltages above $350 \mathrm{~V}$, this gain diverges from its initial behaviour due to optical positive feedback as a result of the additional xenon scintillation produced in the electron avalanche processes. These photons will eject additional photoelectrons from the CsI photocathode, resulting in a faster increase of gain.

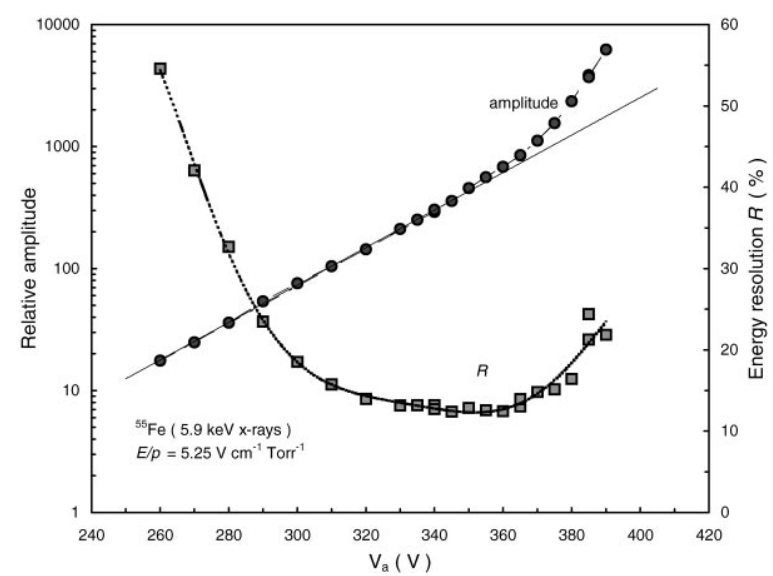

Fig. 5. GPSC/CsI-MSP relative pulse amplitude and energy resolution as a function of the anode-to-cathode strips voltage, $V_{\mathrm{a}}$, for $5.9 \mathrm{keV} \mathrm{X-rays} \mathrm{and} \mathrm{for} \mathrm{a} \mathrm{constant} \mathrm{reduced} \mathrm{electric} \mathrm{field} \mathrm{in}$ the scintillation region $\left(E / p=5.25 \mathrm{~V} \mathrm{~cm}^{-1} \mathrm{Torr}^{-1}\right)$.
In Fig. 6 we present the relative gain due to positive feedback, $G_{\text {tot }} / G_{\text {ex }}$, where $G_{\text {tot }}$ is the measured total gain of the detector and $G_{\text {ex }}$ is the gain represented by the exponential function (Fig. 5). For comparison purposes this feedback gain is presented for the same MSP and for two cases: MSP operating with and without the CsI film deposited on its surface. As seen, the positive feedback due to the presence of the CsI is revealed.

The same trend of Figs. 5 and 6 is observed for 22.1 and $59.6 \mathrm{keV}$ X-rays. This shows that positive feedback does not depend on the number of primary electrons but only on the individual electron avalanche gain.

Photocathode thicknesses of 50, 250 and $500 \mathrm{~nm}$ were studied and they have led to similar results.

Typical pulse-height distributions for ${ }^{55} \mathrm{Fe}$, ${ }^{109} \mathrm{Cd}$ and ${ }^{241} \mathrm{Am}$ radioactive sources are presented in Fig. 7. The ${ }^{55} \mathrm{Fe} \mathrm{X}$-rays were filtered with a chromium film to remove the $\mathrm{Mn} \mathrm{K}_{\beta}$ line and show an energy resolution of $12.5 \%$. As seen in the ${ }^{55} \mathrm{Fe}$ pulse-height distribution, the detector electric noise tail in the low-energy limit is below $250 \mathrm{eV}$. The spectral features of ${ }^{241} \mathrm{Am}$ include the full energy peak at $59.6 \mathrm{keV}$ and the $\mathrm{K}_{\alpha}$ and $\mathrm{K}_{\beta}$ xenon fluorescence escape peaks and the $\mathrm{L} \mathrm{X}$-rays lines from neptunium. All three pulse-height distributions exhibit low background levels.

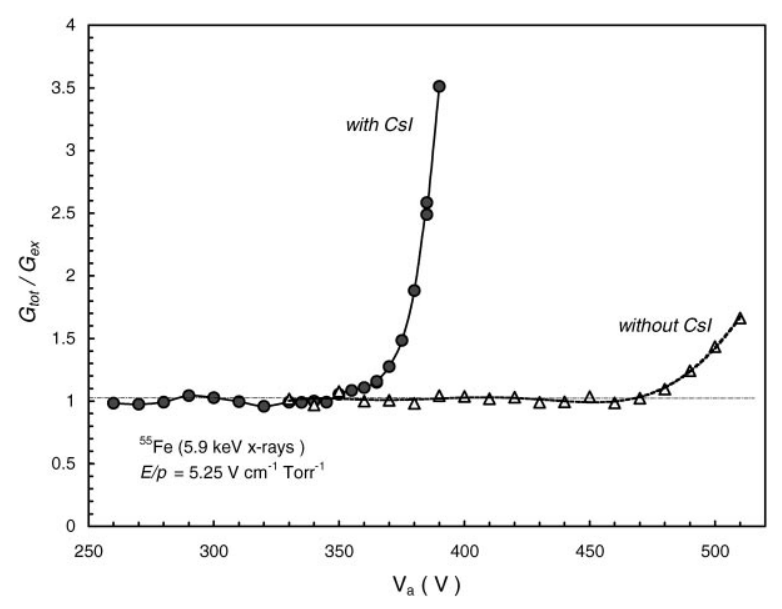

Fig. 6. Positive feedback gain as a function of the anode-tocathode strips voltage, $V_{\mathrm{a}}$, for the detector with a MSP operating with CsI and without CsI in a xenon environment. 

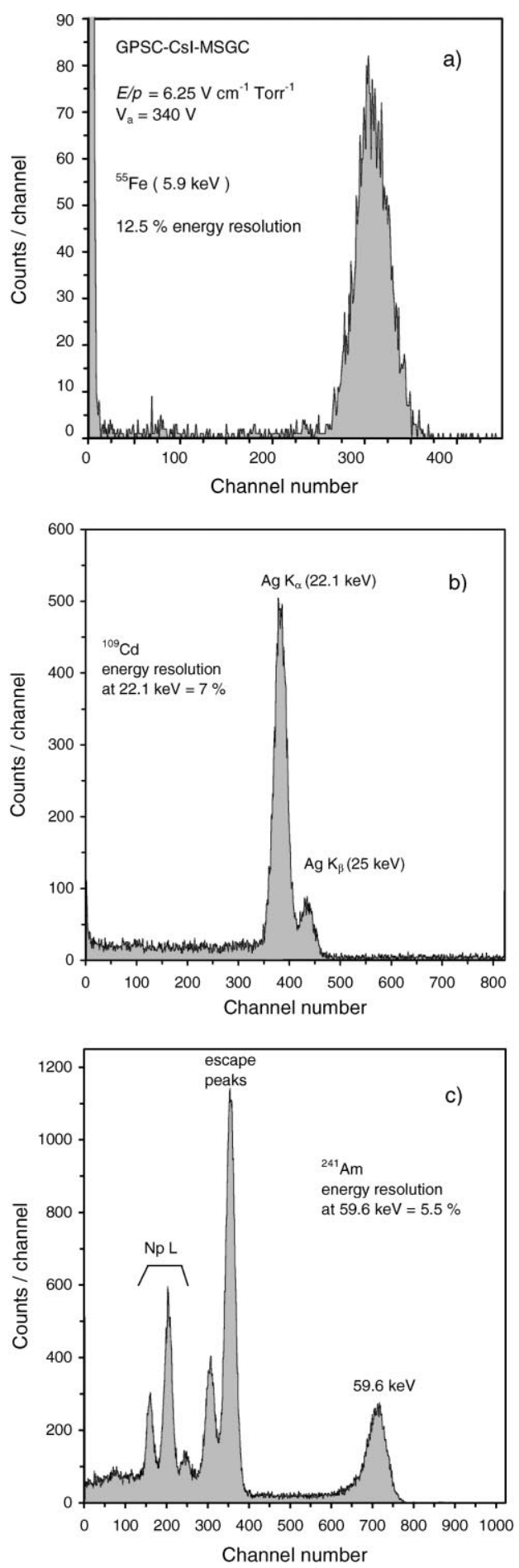

Fig. 7. Typical pulse-height distributions for: (a) $5.9 \mathrm{keV} \mathrm{X-rays;}$ (b) ${ }^{109} \mathrm{Cd}$ X-ray source; (c) ${ }^{241} \mathrm{Am}$ source, of the GPSC instrumented with an integrated CsI-covered MSP.
We have studied the ageing of the CsI photocathode under the influence of the Xe VUV scintillation at a $V_{\mathrm{a}}$ of $360 \mathrm{~V}$. We used $59.6 \mathrm{keV} \mathrm{X-rays} \mathrm{to}$ produce a VUV flux of $\sim 10^{8}$ photons $\mathrm{s}^{-1} \mathrm{~cm}^{-2}$. For constant electric fields in the absorption and scintillation regions and for constant $V_{\mathrm{a}}$ voltages, the relative variation of the $59.6 \mathrm{keV}$ peak amplitude is equal to the relative variation of the photocathode quantum efficiency. Fig. 8 depicts the obtained variation of the relative quantum efficiency as a function of the total surface charge density delivered by the CsI. As shown, after an initial reduction of about $15 \%$ in the relative quantum efficiency with the surface charge density delivered by the CsI (up to about $4 \mu \mathrm{C} \mathrm{mm}^{-2}$ ), only a small variation occurs when the delivered charge increases up to $15 \mu \mathrm{Cmm}^{-2}$. The results show the good behaviour of the CsI photocathode ageing under these experimental conditions. Similar behaviour was described in Ref. [16].

A driftless GPSC with a $8.5 \mathrm{~mm}$ deep scintillation region and using a CsI-covered microstrip plate within the xenon envelope has been built for detection of low-energy X-rays $(\sim 2 \mathrm{keV})$ under a strong magnetic field (5 T), achieving energy resolutions of $23 \%$ for $1.74 \mathrm{keV} \mathrm{X-rays} \mathrm{[35].} \mathrm{These}$ values, although worse than the ones obtained with

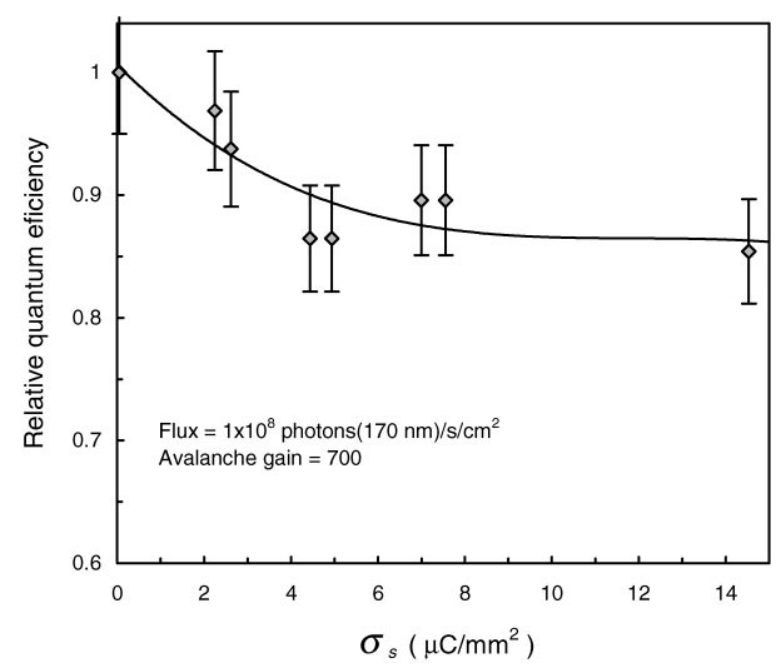

Fig. 8. Relative detector pulse amplitude variation as a function of the surface charge density, $\sigma_{\mathrm{s}}$, delivered by the CsI photocathode. 
a PMT-based GPSC ( 14\%) [36], are better than the ones obtained with a Xe-filled standard MSGC $(\sim 30 \%)$ [37].

\section{GPSC coupled to a separated MSGC photosensor}

The GPSC can be coupled to a separated MSGC photosensor using the CsI-covered MSP in a P10 atmosphere. Such a detector was described in Ref. [11] and is more complex and more difficult to operate. On the other hand, it presents improved performance relative to the one instrumented with an integrated photosensor. The use of a quenching gas in the photosensor reduces the effect of positive feedback and $V_{\text {a }}$ voltages up to $460 \mathrm{~V}$ can be used without the occurrence of noticeable positive feedback. Also, as the GPSC scintillation region is separated from the photosensor by a quartz window, the electric field in the region above the MSP can be kept low enough to avoid the saturation of detector pulse amplitudes, at high values of $E / p$ in the scintillation region. For this detector, the characteristic behaviour of GPSC amplitude variation with $E / p$ in the scintillation region is observed for

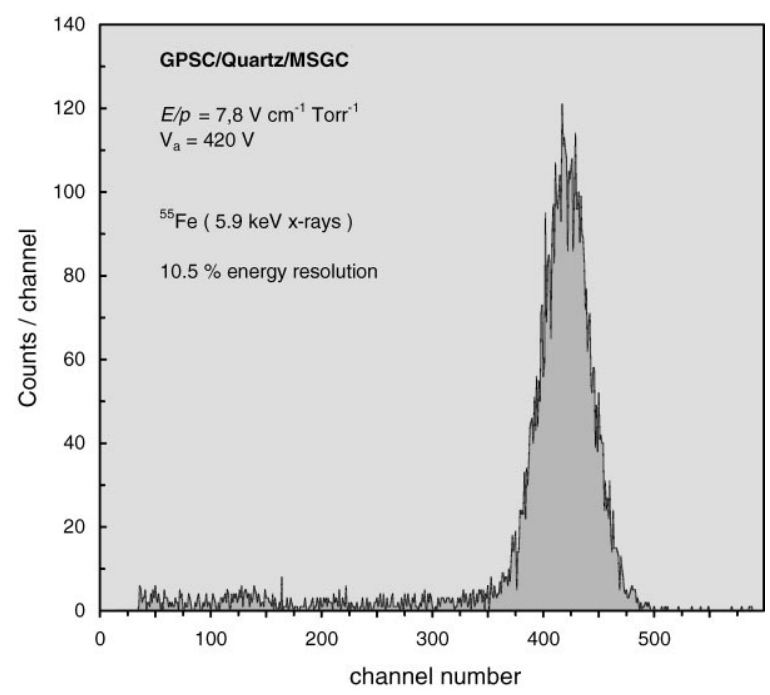

Fig. 9. Typical pulse-height distribution for $5.9 \mathrm{keV}$ X-rays of the xenon-filled GPSC coupled to a P10filled MSGC photosensor through a quartz window. the whole $E / p$ range (e.g. as detailed in Ref. [30]). As a consequence of the mentioned improvements, this detector presented an energy resolution of $11.5 \%$ for $5.9 \mathrm{keV}$ X-rays [11].

Using the improved cleaning and handling procedures for the MSP and CsI photocathode preparation the energy resolution improved to $10.5 \%$ for $5.9 \mathrm{keV}$ X-rays (Fig. 9) and the energy noise tail decreased to values below $60 \mathrm{eV}$. This performance is somewhat better than the one achieved when using an integrated CsI-covered MSP photosensor.

\section{Conclusions}

It was shown that a CsI-covered MSP is an attractive alternative to PMT as an integrated photosensor of GPSCs in applications where cost, compactness, area, and power consumption are important criteria, with the possibility of working at higher pressures than the ones for standard GPSCs. Detector energy resolutions of $12 \%, 7 \%$ and $5.5 \%$ were obtained for $5.9,22.1$ and $59.6 \mathrm{keV}$, respectively. These values are already better than the ones achievable with standard proportional counters or MSGCs.

The importance of the cleaning and handling procedures for the MSP and CsI photocathode preparation should be stressed. Namely, the heating under vacuum of the CsI photocathode film prior to operation, for removal of water contamination, is one of the most important factors to achieve improved photosensor performance.

Further studies to improve the performance of this integrated photosensor can be investigated. The use of MSPs with larger areas and larger photocathode-strips-area-to-total-MSP-area ratio may increase the number of photoelectrons produced per primary electron. Also, increasing the distance between the anode and photocathode strips as well as reducing the anode strip width, could lead to a higher photosensor gain with lower positive feedback.

The performance achieved with a GPSC coupled to an independent MSGC photosensor using the CsI-covered MSP in a P10 atmosphere is somewhat better than the one achieved with a GPSC using an integrated CsI-covered MSP. A GPSC 
energy resolution of $10.5 \%$ is achieved for $5.9 \mathrm{keV}$ $\mathrm{X}$-rays when such an independent photosensor is used.

\section{Acknowledgements}

The work was carried out in the Atomic and Nuclear Instrumentation Group of the Instrumentation Centre (Unit 217/94) of Departamento de Física, Universidade de Coimbra. Support is acknowledged from project CERN/P/FIS/15200/99. Thanks are due to C.M.B. Monteiro for article style revising.

\section{References}

[1] C.A.N. Conde, A.J.P.L. Policarpo, Nucl. Instr. and Meth. 53 (1967) 7.

[2] A.J.P.L. Policarpo, Space Sci. Instr. 3 (1977) 77.

[3] H.E.N. Ngoc, J. Jeanjean, H. Itoh, G. Charpack, Nucl. Instr. and Meth. 172 (1980) 603.

[4] A. Peacock, B.G. Taylor, N. White, T. Courvoisier, G. Manzo, IEEE Trans. Nucl. Sci. 32 (1985) 108.

[5] Varvaritsa, I.V. Vikulov, V.V. Ivashov, M.A. Panov, V.I. Filatov, K.I. Schekin, Instr. Exp. Tech. 35 (1992) 745.

[6] A.J.P.L. Policarpo, Nucl. Instr. and Meth. 153 (1978) 389.

[7] D.F. Anderson, Nucl. Instr. and Meth. 178 (1980) 125.

[8] J.C. Van Standen, J. Foh, M. Mutterer, J. Pannicke, K.P. Schelhaas, J.P. Theobald, Nucl. Instr. and Meth. 157 (1978) 301.

[9] A.J. de Campos, IEEE Trans. Nucl. Sci. NS-31 (1984) 133.

[10] D.G. Simons, P.A.J. Korte, A. Peacock, A. Smith, J.A.M. Bleeker, IEEE Trans. Nucl. Sci. NS-32 (1985) 345.

[11] J.F.C.A. Veloso, J.M.F. dos Santos, C.A.N. Conde, Nucl. Instr. and Meth. A 422 (1999) 273.

[12] V. Dangendorf, A. Breskin, R. Chechik, H. Schmidt-Bocking, Nucl. Instr. and Meth. A 289 (1990) 322.

[13] J.F.C.A. Veloso, J.A.M. Lopes, J.M.F. dos Santos, C.A.N. Conde, IEEE Trans. Nucl. Sci. 43 (1996) 1232.

[14] J.A.M. Lopes, J.M.F. dos Santos, C.A.N. Conde, R.E. Morgado, IEEE Trans. Nucl. Sci. 44 (1997) 517.

[15] F. Piuz, Nucl. Instr. and Meth. A 371 (1996) 96.

[16] J. Vávra, Nucl. Instr. and Meth. A 371 (1996) 33.

[17] J. Seguinot, T. Ypsilantis, J.P. Jobez, R. Arnold, J.L. Guyonnet, E. Chesi, J. Tischhauser, R.J. Mountain, I.
Adachi, T. Sumiyoshi, Nucl. Instr. and Meth. A 371 (1996) 64.

[18] K. Zeitelhack, J. Friese, R. Gernhäuser, P. Kienkle, H.-J. Körner, P. Maier-Komor, S. Winkler, Nucl. Instr. and Meth. A 351 (1994) 585.

[19] F. Angelini, R. Bellazzini, L. Bosisio, A. Brez, M.M. Massai, G. Spandre, M.R. Torquati, Nucl. Instr. and Meth. A 371 (1996) 358.

[20] A. Oed, Nucl. Instr. and Meth. A 263 (1988) 351.

[21] R. Bellazzini, M.A. Spezziga, Rivista del Nuovo Cimento 17 (12) (1994) 1.

[22] D.G. Simons, P.A.J. De Korte, Nucl. Instr. and Meth. A 277 (1989) 642.

[23] J. Miyamoto, G.F. Knoll, Nucl. Instr. and Meth. A 399 (1997) 85.

[24] G.F. Knoll, Radiation Detection and Measurement, Second Edition, Wiley, New York, 1989, pp. 174-178.

[25] T.H.V.T. Dias, J.M.F. dos Santos, P.J.B.M. Rachinhas, F.P. Santos, C.A.N. Conde, A.D. Stauffer, J. Appl. Phys. 82 (1997) 2742.

[26] G.D. Alkazov, A.P. Komar, A.A. Vorob'ev, Nucl. Instr. and Meth. 48 (1967) 1.

[27] D.F. Anderson, S. Kwan, V. Peskov, B. Hoeneisen, Nucl. Instr. and Meth. A 323 (1992) 626.

[28] A. Breskin, A. Buzulutskov, R. Chechik, IEEE Trans. Nucl. Sci. NS-42 (1995) 298.

[29] J.E. Lees, G.W. Fraser, S.E. Pearce, J.F. Pearson, V.N. Shchemelev, A.P. Pavlov, A.S. Shulakov, Nucl. Instr. and Meth. A 381 (1996) 453.

[30] F.I.G.M. Borges, J.M.F. dos Santos, T.H.V.T. Dias, F.P. Santos, P.J.B.M. Rachinhas, C.A.N. Conde, Nucl. Instr. and Meth. A 422 (1999) 321.

[31] F.I.G.M. Borges, C.A.N. Conde, Electron transport and grid transmission in noble gas radiation detectors, in: T.D. Märk, R. Schrittwieser, D. Smith (Eds.), Proceedings of the SASP-94, Symposium on Atomic Cluster and Surface Physics, Maria Alm-Intermoos, Austria, 20-26 March 1994, p. 436.

[32] A. Di Mauro, E. Nappi, F. Posa, A. Breskin, A. Buzulutskov, R. Chechik, S.F. Biagi, G. Paic, F. Piuz, Nucl. Instr. and Meth. A 371 (1996) 137.

[33] F. Hartjes, B. Hendriksen, Nucl. Instr. and Meth. A 310 (1991) 88.

[34] Z.Q. Yu, S.S. Wang, H.J. Wei, M.L. Chu, P.K. Teng, Nucl. Instr. and Meth. A 372 (1996) 35.

[35] J.F.C.A. Veloso, J.M.F. dos Santos, C.A.N. Conde, Nucl. Instr. and Meth. A (2001) in press.

[36] J.M.F. dos Santos, A.C.S.S.M. Bento, C.A.N. Conde, Nucl. Instr. and Meth. A 337 (1994) 427.

[37] J.F.C.A. Veloso, J.M.F. dos Santos, C.A.N. Conde, R.E. Morgado, X-ray Spectrom. 26 (1997) 237. 